\title{
Scleral Lens Issues and Complications: Their Recognition, Etiology, and Management
}

\author{
Author: Daddi Fadel DOptom, FSLS, FBCLA \\ Published 2020 by Dougmar Publishing Group Inc., Dundas, ON; 386 pages.
}

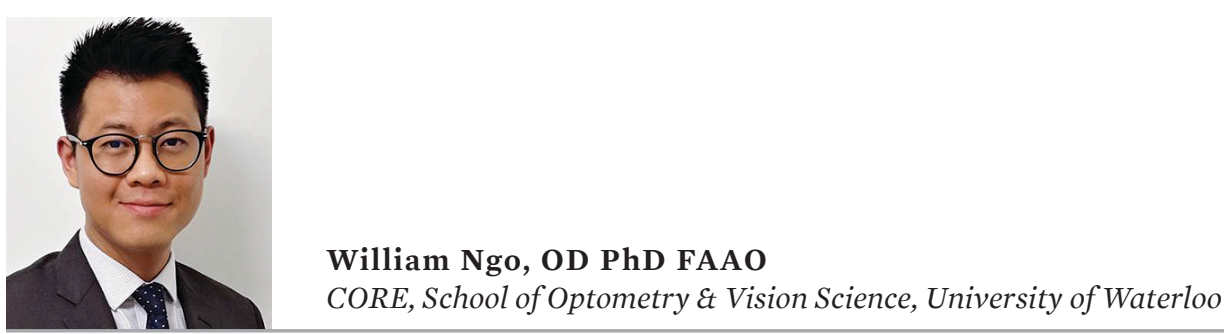

cleral lenses are typically used to manage complicated corneal conditions and ocular surface diseases. Up until the recent decade, the fitting and prescribing of scleral lenses belonged to very niche groups in optometry. However, over time, the advancement in gas permeable materials, manufacturing, and fitting techniques has led to a surge in popularity in fitting scleral lenses. This increase in accessibility has enabled more clinicians to take on and manage more complicated corneal and ocular surface diseases, directly translating into greater patient benefit and care. Today, the art and science of scleral lens fitting is evolving faster than ever.

Despite the popularity of scleral lenses, the practice of fitting scleral lenses can still be intimidating. The amount of time that could be spent troubleshooting fits and managing complications can be frustrating and costly for both the practitioner and patient. This book, Scleral Lens Issues and Complications: Their Recognition, Etiology, and Management, is a timely publication that combines scientific evidence and clinical pearls, covering a wide range of topics related to scleral lens complications.

The first two chapters of the book discusses how vital dyes (fluorescein and lissamine green) are used to assess and diagnose the quality of scleral lens fits and its relationship to the scleral profile. The text is supplemented by high quality illustrations demonstrating the concepts described.

The next two chapters, forming the bulk of the book, discusses the issues and complications related to scleral lens fitting and wear. This section is presented systematically for each condition; starting with the examination technique (e.g., slit lamp illumination level, illumination technique, magnification), followed by symptoms and signs, etiology and management. The writing in this section is highly abbreviated but effective, making it suitable for quick referencing. These issues and complications, including those related to poor lens fitting, lens handling, care, and compliance are summarized in the subsequent chapter.

Following this, the next section discusses factors that affect vision and comfort. This chapter is valuable for troubleshooting, covering nearly 30 different situations where comfort and or vision are altered with scleral lens wear (e.g., midday fogging, poor wettability, residual ocular astigmatism). These conditions are also summarized in tables for easy referencing. Related to optics, the next chapter discusses various front surface optics that are available to scleral lenses, such as toric correction for astigmatism, multifocals for presbyopia, wavefront-guided designs to manage aberrations, and prism for binocular vision correction. Finally, the book concludes with a section on scleral lens cleaning, disinfection processes, handling, and storage cases for various types of scleral lenses. This chapter is helpful for highlighting the important points that should be conveyed to the patient.

Overall, this book summarizes nearly all the issues and complications one would encounter with fitting scleral lenses. It will serve as a helpful resource for a wide range of practitioners, whether it be those getting started with fitting scleral lenses, or those who are more experienced with scleral lenses and simply want a book for quick referencing. 